\title{
12/15 lipoxygenase regulation of colorectal tumorigenesis is determined by the relative tumor levels of its metabolite 12- HETE and 13-HODE in animal models
}

\author{
Jian Chang ${ }^{1,3, *}$, Li Jiang 1,4,*, Yinqiu Wang ${ }^{1}$, Bing Yao', Shilin Yang ${ }^{1}$, Bixiang Zhang ${ }^{3}$ \\ and Ming-Zhi Zhang ${ }^{1,2,5}$ \\ ${ }^{1}$ Department of Medicine, Vanderbilt University School of Medicine, Nashville, TN, USA \\ ${ }^{2}$ Cancer Biology, Vanderbilt University School of Medicine, Nashville, TN, USA \\ ${ }^{3}$ Hepatic Surgery Center,Tongji Hospital, Tongji Medical College, Huazhong University of Science \& Technology, Wuhan, china \\ ${ }^{4}$ Department of Biliary and Pancreatic Surgery, Tongji Hospital, Tongji Medical College, Huazhong University of Science \& \\ Technology, Wuhan, china \\ ${ }^{5}$ Jiangsu Center for the Collaboration and Innovation of Cancer Biotherapy, Cancer Institute, Xuzhou Medical College, \\ Xuzhou, China \\ * These authors contributed equally to this work \\ Correspondence to: Ming-Zhi Zhang, email: ming-zhi.zhang@vanderbilt.edu \\ Keywords: arachidonic acid, linoleic acid, metabolites, epithelial cell, stroma, mice \\ Received: August 04, $2014 \quad$ Accepted: December 12, $2014 \quad$ Published: December 18, 2014
}

This is an open-access article distributed under the terms of the Creative Commons Attribution License, which permits unrestricted use, distribution, and reproduction in any medium, provided the original author and source are credited.

\section{ABSTRACT}

Colorectal cancer (CRC) continues to be a major cause of morbidity and mortality. The arachidonic acid (AA) pathway and linoleic acid (LA) pathway have been implicated as important contributors to CRC development and growth. Human 15-lipoxygenase 1 (15-LOX-1) converts LA to anti-tumor 13-S-hydroxyoctadecadienoic acid (13-HODE) and 15-LOX-2 converts AA to 15-hydroxyeicosatetraenoic acid (15-HETE). In addition, human 12-LOX metabolizes AA to pro-tumor 12-HETE. In rodents, the function of 12LOX and 15-LOX-1 and 15-LOX-2 is carried out by a single enzyme, 12/15-LOX. As a result, conflicting conclusions concerning the role of 12-LOX and 15-LOX have been obtained in animal studies. In the present studies, we determined that PD146176, a selective 15-LOX-1 inhibitor, markedly suppressed 13-HODE generation in human colon cancer HCA-7 cells and HCA-7 tumors, in association with increased tumor growth. In contrast, PD146176 treatment led to decreases in 12-HETE generation in mouse colon cancer MC38 cells and MC38 tumors, in association with tumor inhibition. Surprisingly, deletion of host 12/15-LOX alone led to increased MC38 tumor growth, in association with decreased tumor 13-HODE levels, possibly due to inhibition of $12 / 15-$ LOX activity in stroma. Therefore, the effect of 12/15-LOX on colorectal tumorigenesis in mouse models could be affected by tumor cell type (human or mouse), relative $12 / 15$ LOX activity in tumor cells and stroma as well as the relative tumor 13-HODE and 12-HETE levels.

\section{INTRODUCTION}

Arachidonic acid (AA), a polyunsaturated omega- 6 fatty acid, is a component of the phospholipid domain of most cell membranes[1]. AA is cleaved from membrane phospholipids by cytosolic phospholipase $\mathrm{A}_{2}$ and metabolized to eicosanoids by three main pathways: the cyclooxygenase (COX) pathway, the lipoxygenase (LOX) pathway, and the cytochrome P450 pathway. The cyclooxygenase pathway generates prostanoids, including prostaglandins (PGEs) and thromboxane; the lipoxygenase pathway produces leukotrienes and hydroxyeicosatetraenoic acids (HETEs). In the cytochrome P450 pathway, AA is metabolized to epoxyeicosatrienoic acids and HETEs by cytochrome P450 epoxygenases and 
cytochrome P450 $\omega$-hydroxylases, respectively.

Colorectal cancer (CRC) is one of the most preventable cancers; however it is still the leading cause of cancer death. The arachidonic acid pathway has been implicated as an important contributor to CRC development and growth. Arachidonic acid metabolites derived from the cyclooxygenase, the LOX or the cytochrome P450 pathway have all been implicated in colorectal tumorigenesis[2-4]. COX-derived $\mathrm{PGE}_{2}$ is the major prostanoids to promote colorectal tumorigenesis[5]. However, both 5-LOX-derived leukotrienes and 5-HETE have also been implicated in colorectal tumorigenesis[6-10]. Either genetic or pharmacologic inhibition of 5-LOX pathway suppresses colorectal tumorigenesis in murine models[3, 8-11].

In addition to 5-LOX, humans have other two major LOX isoforms: 12-LOX, and 15-LOX. 12-LOX and its metabolite 12-HETE have been reported to promote tumorigenesis and metastasis by inhibiting apoptosis, stimulating cell proliferation, angiogenesis, interactions between tumor cell and vasculature, tumor cell mobility, invasion and proteolysis[12]. Polymorphism (Arg261Gln) in the 12-LOX gene has been associated with a reduced risk of colorectal adenoma[13]. In humans, 15-LOX exists as two isozymes, 15-LOX-1 and 15-LOX-2. The preferred substrate for 15-LOX-1 and 15-LOX2 are linoleic acid and arachidonic acid, respectively. 15-LOX-1 and its linoleic acid metabolite, 13-HODE (13-S-hydroxyoctadecadienoic acid), are decreased in human colon cancer[14, 15]. 13-S-HODE inhibits cell proliferation and induces apoptosis in colon cancer cells[16].

In rodents, there are not separate $12-\mathrm{LOX}$ and 15-LOX enzymes. Instead, 12/15-LOX in rodents functions as both 12-LOX and 15-LOX. Rodent 12/15LOX can metabolizes arachidonic acid to 12-HETE and 15-HETE and linoleic acid to 13-HODE. Due to this special property of $12 / 15$-LOX in rodents, information about its role in tumorigenesis in mouse models is limited and controversial. 12/15-LOX has been reported to promote prostate carcinoma progression[17] and to mediate invasion of intrametastatic lymphatic vessels and propagate lymph node metastasis in a mouse model of mammary carcinoma[18]. However, overexpression of $15-\mathrm{LOX}-1$ in intestinal epithelial cells inhibited colonic tumorigenesis in mice[19]. Using mouse and human colorectal tumor models as well as genetic and pharmacologic inhibition of LOX activity, we determined that the effect of inhibition of LOX activity on colorectal tumorigenesis depends on LOX-derived metabolites in both the tumor cells and the host stroma.

\section{RESULTS}

\section{Pharmacologic or genetic inhibition of LOX activity had differential effects on murine and human colorectal tumorigenesis}

The effect of 15-LOX on tumorigenesis is limited and controversial[17-19]. To investigate the potential role of 15-LOX in mouse and human colorectal tumorigenesis, mouse colon adenocarcinoma MC38 cells and human colon adenocarcinoma HCA-7 cells were used for mouse and human colonic tumor growth experiments. Treatment with a commercially available 15-LOX-1 inhibitor, PD146176, markedly inhibited mouse MC38 tumor growth $(123 \pm 24$ vs. $227 \pm 40 \mathrm{mg} /$ mouse of vehicle, $P<$ $0.05, \mathrm{n}=10$ ) (Figure 1A). In contrast, PD146176 treatment promoted human HCA-7 tumor growth (493 \pm 62 vs. 217 $\pm 27 \mathrm{mg} /$ mouse of vehicle, $P<0.001, \mathrm{n}=6)$ (Figure $2 \mathrm{~A}$ ).

Administration of PD146176 inhibits both tumor cell and host 15-LOX activity. To investigate the potential role of host $15-\mathrm{LOX}$ in colorectal tumorigenesis, C57BL/6 wild type or 12/15-LOX knockout mice were subcutaneously injected with MC38 cells and tumor size was evaluated 18 days later. As indicated in Figure 1A, deletion of host $12 / 15$-LOX led to increases in mouse MC38 tumor growth $(458 \pm 99$ vs. $227 \pm 40 \mathrm{mg} /$ mouse of vehicle, $P<0.05, \mathrm{n}=10)$, opposite to that seen with PD146176 treatment.

\section{Eicosanoid profiling in mouse MC38 tumors and human HCA-7 tumors}

To investigate the potential mechanisms underlying the differential effects of 15-LOX inhibition on mouse and human colorectal tumorigenesis, we evaluated tumor eicosanoid profiling by gas chromatographic/ negative ion chemical ionization mass spectrometric assays using stable isotope dilution[20]. As shown in Figure 1B and supplemental Figure S1A, 12-HETE was the major arachidonic acid metabolite in MC38 tumors, and its production was markedly inhibited by PD146176 treatment $(1498 \pm 250$ vs. $3150 \pm 566 \mathrm{ng} / \mathrm{gram}$ tissue of vehicle, $P<0.05, \mathrm{n}=4$ ), but not by deletion of host $12 / 15$ LOX. No other arachidonic acid-derived metabolites were affected by PD146176 treatment. In addition to arachidonic acid-derived metabolites, MC38 tumors also had high levels of 13-HODE, a linoleic acid-derived metabolite catalyzed by the human 15-LOX-1 enzymatic activity or mouse 12/15-LOX enzymatic activity. The production of 13-HODE in MC38 tumors was markedly inhibited by either PD146176 administration or deletion of host 12/15-LOX (ng/gram tissue: wild type: $2441 \pm 212$; PD146176: $1412 \pm 130, P<0.01 ; 12 / 15-\mathrm{LOX}$ deletion: $1577 \pm 87, P<0.01 . \mathrm{N}=4$ in all groups). These results 
suggest that both 12-HETE and 13-HODE might regulate MC38 tumor growth.

In human HCA-7 tumors, 12-HETE and 13-HODE were the major metabolites of arachidonic acid and linoleic acid (Figure 2B and supplemental Figure S1B). Among all evaluated metabolites from arachidonic acid and linoleic acid in HCA-7 tumors, only the production of 13-HODE was inhibited by PD146176 treatment, suggesting that decreased 13-HODE production might contribute to increased HCA-7 tumor growth in PD146176 treated mice.

\section{2/15-LOX enzymatic activity contributed to 13 - HODE production in mouse macrophage}

In the subcutaneous Xenograft tumors, metabolites of both arachidonic acid and linoleic acid might come from tumor cells or stromal cells (cells from host, including infiltrating immune cells, particularly macrophages). It has been reported that $12 / 15$-LOX is primarily expressed in macrophages in mice[21]. To investigate the potential contribution of macrophages to tumor eicosanoid

A
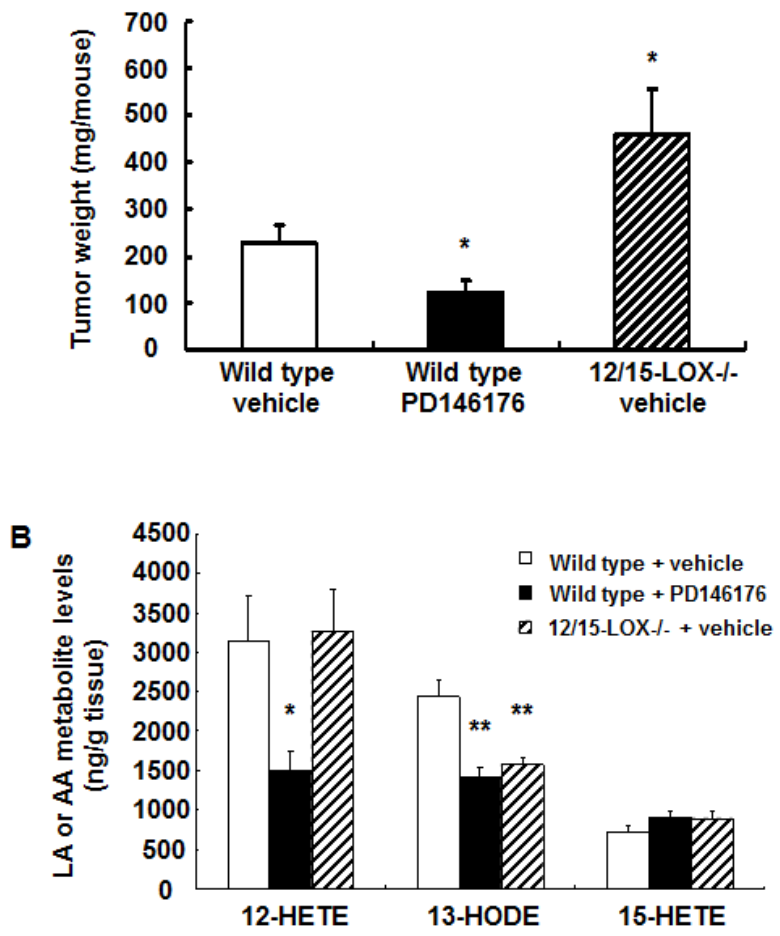

Figure 1: Effects of pharmacologic and genetic inhibition of LOX activity on mouse colonic MC38 tumor growth and tumor eicosanoid production. A. Inhibition of LOX activity with PD146176 inhibited, while host 12/15-LOX deletion promoted, MC38 tumor growth $(* P<$ 0.05 vs. wild type vehicle, $\mathrm{n}=10$ in each group). B. PD146176 treatment inhibited MC38 tumor production of both 12-HETE and 13-HODE while host 12/15-LOX deletion only inhibited 13 -HODE production ( $* P<0.05$ and $* * P<0.01$ vs. wild type vehicle, $\mathrm{n}=4$ in each group). production, intestinal macrophages from C56BL/6 mice were isolated using CD11b beads, incubated with substrate (60 $\mu \mathrm{M}$ arachidonic acid or $60 \mu \mathrm{M}$ linoleic acid, $60 \mathrm{~min}$ ), and metabolites were measured. Enzyme activity was expressed as $\mathrm{pM} / \mathrm{min} / \mathrm{mg}$ protein. As indicated in Figure $3 \mathrm{~A}$, macrophages produced similar high levels of 12 HETE, 15-HETE and 15-HODE, all of which could be the products of murine 12/15-LOX. Macrophages also produced 5-HETE and $\mathrm{PGE}_{2}$ (Figure 3B). Surprisingly, deletion of 12/15-LOX only significantly inhibited 13-HODE production (pM/min/mg: wild type: $39.3 \pm$ 2.1 ; 12/15-LOX knockout: $24.9 \pm 1.1, P<0.01, \mathrm{n}=3$ ), indicating that macrophage $12 / 15$-LOX has the enzymatic activity converting linoleic acid to 13 -HODE.

PD146176 inhibited 13-HODE production in HCA-7 cells and decreased 12-HETE generation in MC38 cells

To investigate the effect of PD146176 on the production of metabolites from arachidonic acid or linoleic acid, MC38 cells and HCA-7 cells were incubated with substrate (arachidonic acid or linoleic acid) with or without the presence of PD146176. As indicated in Figure 4, PD146176 inhibited 12-HETE production in MC38

A

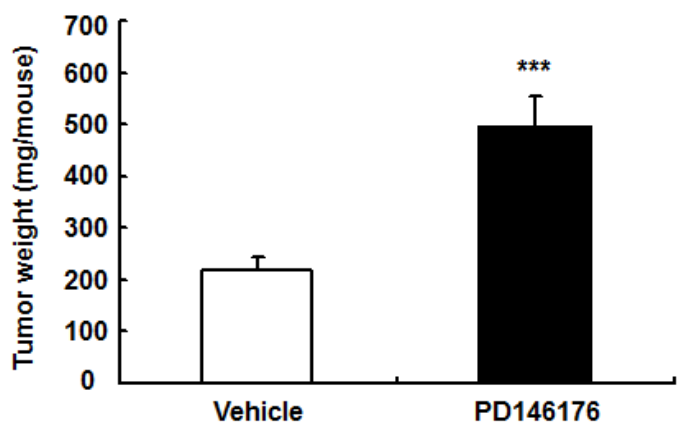

B

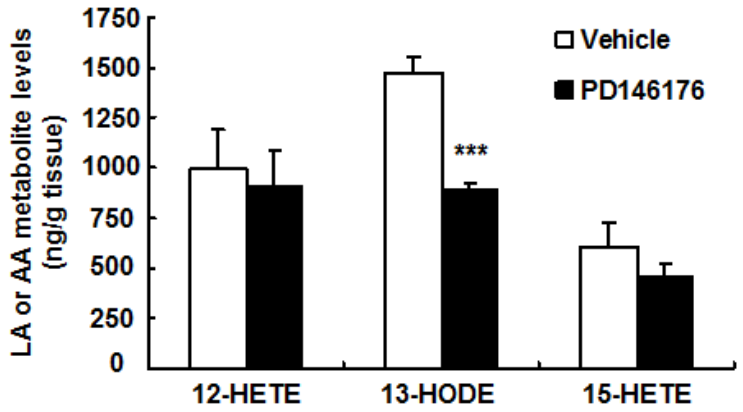

Figure 2: Inhibition of LOX activity with PD146176 led to increased human colonic HCA-7 tumor growth and decreased tumor 13-HODE production. A. PD146176 treatment led to increased HCA-7 tumor growth $(* * * P<0.001$ vs. vehicle, $\mathrm{n}=6$ in each group). B. PD146176 treatment led to decreased 13-HODE production in HCA-7 tumor $(* * * P<$ 0.001 vs. vehicle, $\mathrm{n}=4$ in each group). 
cells $(12.37 \pm 0.82 \mathrm{vs} .19 .45 \pm 0.60 \mathrm{pM} / \mathrm{min} / \mathrm{mg}$ of vehicle, $P<0.01, \mathrm{n}=3$ ), but had no effect on the production of 15-HETE and 13-HODE. In contrast, PD146176 inhibited 13-HODE production in HCA-7 cells $(0.09 \pm 0.02$ vs. 0.75 $\pm 0.01 \mathrm{pM} / \mathrm{min} / \mathrm{mg}$ of vehicle, $P<0.001, \mathrm{n}=3$ ), but had no effect on the production of 12-HETE and 15-HETE (Figure $5 \mathrm{~A} \& \mathrm{~B})$.

To further investigate the eicosanoid profiling in mouse MC38 cells metabolized by 12/15-LOX and in human HCA-7 cells by 12-LOX and 15-LOX-1 and 15LOX-2, siRNA technique was used to silence these genes. In MC38 cells, gene silence of Alox15 (12/15-LOX) significantly inhibited the production of 12-HETE (15.27 \pm 1.13 vs. $26.75 \pm 2.26 \mathrm{pM} / \mathrm{min} / \mathrm{mg}$ of mock control, $P<$ $0.001, \mathrm{n}=3)$, but had no effect on the production of 13HODE $(0.414 \pm 0.022$ vs. $0.381 \pm 0.041 \mathrm{pM} / \mathrm{min} / \mathrm{mg}$ of mock control, $P>0.05, \mathrm{n}=3)$ and 15-HETE $(23.74 \pm 3.13$ vs. $25.43 \pm 2.64 \mathrm{pM} / \mathrm{min} / \mathrm{mg}$ of mock control, $P>0.05$, $\mathrm{n}=3$ ). In human HCA-7 cells, gene silence of 15-LOX-1 but not 15-LOX-2 inhibited the production of LA-derived 13-HODE $(0.652 \pm 0.040$ vs. $1.171 \pm 0.088 \mathrm{pM} / \mathrm{min} / \mathrm{mg}$ of mock control, $P<0.001, \mathrm{n}=3$ ), while gene silence of

A

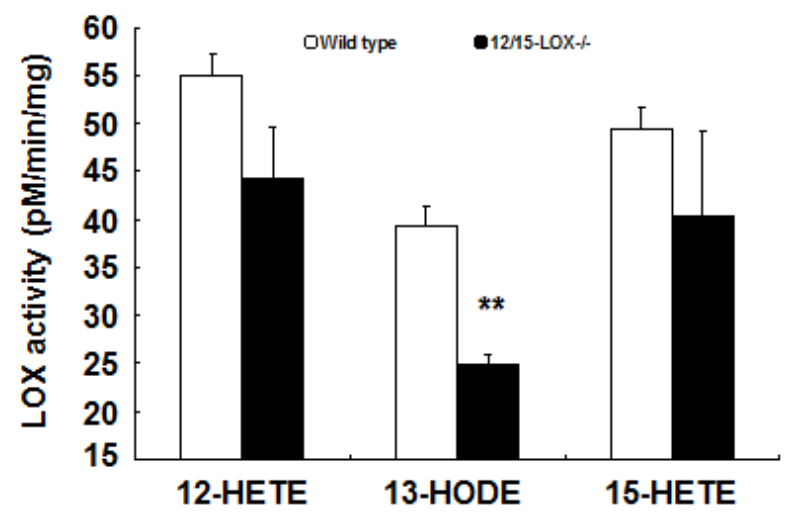

$\mathbf{B}$

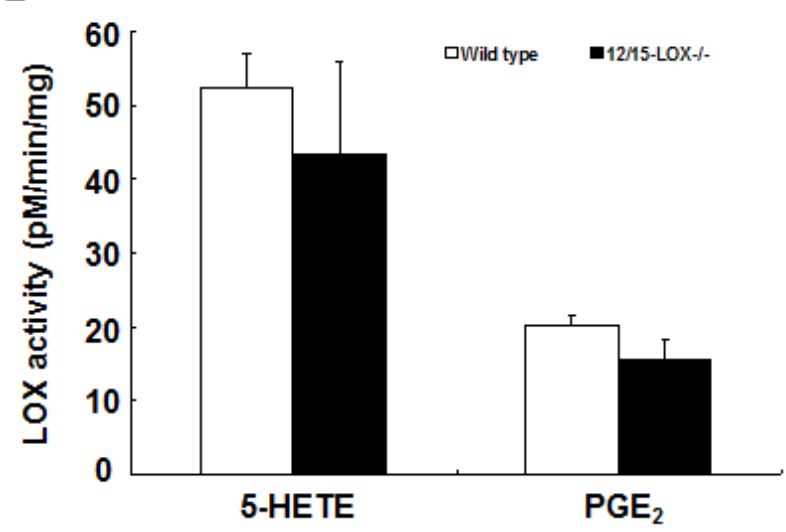

Figure 3: Mouse macrophage 12/15-LOX produced 13-HODE. Mouse intestinal macrophages were isolated with CD11b-beads and their abilities to metabolize arachidonic acid and linoleic acid were investigated. 12/15-LOX deletion led to decreased macrophage production of 13-HODE but had no effect on the production of other eicosanoids $(* * P<0.01$ vs. wild type, $\mathrm{n}=3$ in each group).
15-LOX-2 but not 15-LOX-1 inhibited the production of AA-derived 15 -HETE $(3.620 \pm 0.285$ vs. $6.326 \pm 0.537$ $\mathrm{pM} / \mathrm{min} / \mathrm{mg}$ of Mock control, $P<0.001, \mathrm{n}=3$ ) (Figure 5 C\&D). Gene silence of 12-LOX in HCA-7 cells inhibited the production of AA-derived 12-HETE $(3.583 \pm 0.279$ vs. $5.477 \pm 0.322 \mathrm{pM} / \mathrm{min} / \mathrm{mg}$ of Mock control, $P<0.001$, $\mathrm{n}=3)$.

\section{3-HODE inhibited and 12-HETE stimulated both murine and human colon cancer cell proliferation}

To investigate the direct effects of 12-HETE, 15HETE and 13-HODE on colon cancer proliferation, we treated different mouse and human colon cancer cell lines with these agents and cell proliferation was measured by using cell proliferation assay kit. As shown in Figure 6A, 12-HETE stimulated MC38 cell proliferation at 0.1 $\mu \mathrm{M}$, but stimulated HCA-7 cell proliferation at $10 \mu \mathrm{M}$, indicating that 12-HETE is a more potent mitogen for MC38 cells than HCA-7 cells. In addition, 12-HETE also potently stimulated the proliferation of CT26 cells, another
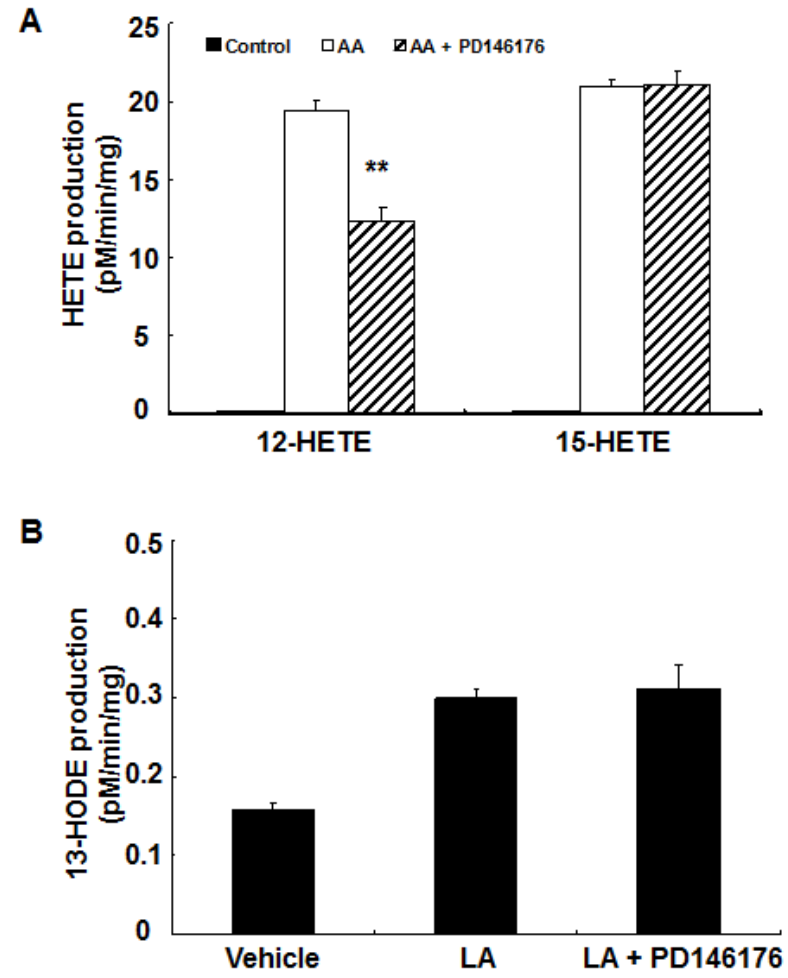

Figure 4: 12-HETE production in MC38 cells was inhibited by PD146176. MC38 cells were incubated with arachidonic acid or linoleic acid with or without PD146176, and medium was harvested for measurement of eicosanoid profiling. Enzymatic activity was expressed as $\mathrm{pM} / \mathrm{min} / \mathrm{mg}$ protein. A. PD146176 inhibited the production of arachidonic acid-derived 12-HETE, but not 15 -HETE ( $* * P<0.01$ vs. vehicle, $\mathrm{n}=3$ in each group). B. PD146176 had no effect on the production of linoleic acid-derived 13-HODE. 
A

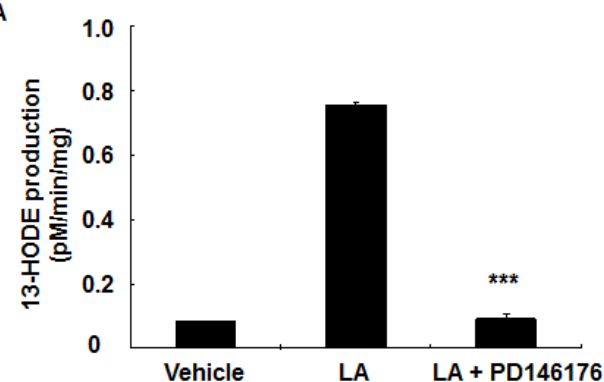

B

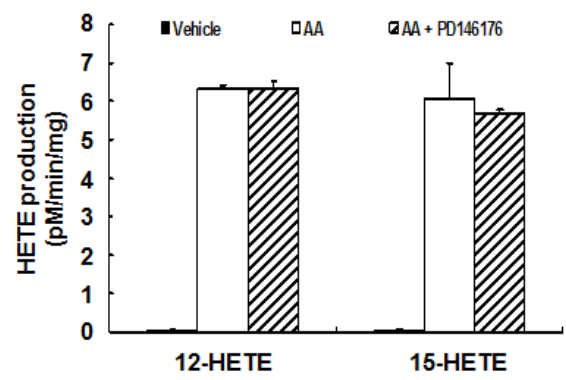

C
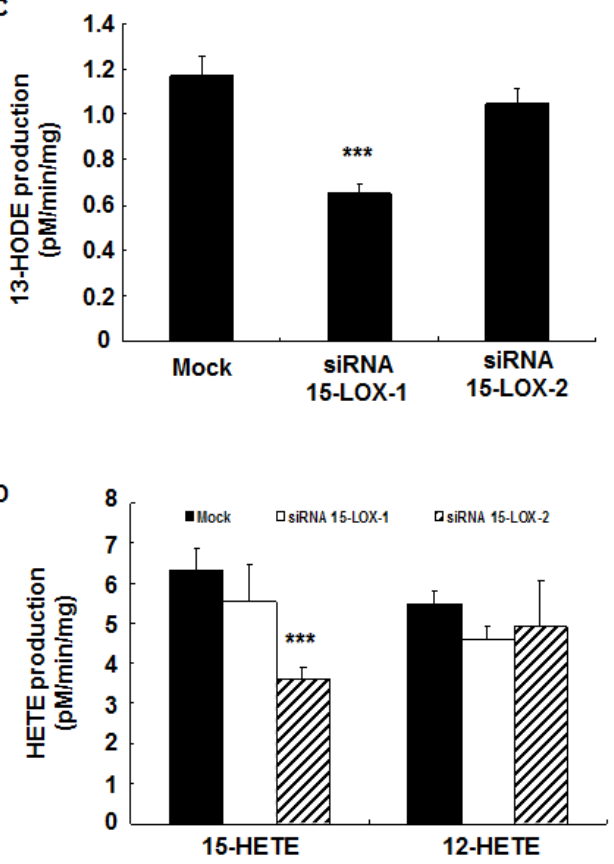

Figure 5: 13-HODE production in HCA-7 cells was inhibited by PD146176. HCA-7 cells were incubated with arachidonic acid or linoleic acid with or without PD146176, and medium was harvested for measurement of eicosanoid profiling. Enzymatic activity was expressed as $\mathrm{pM} / \mathrm{min} / \mathrm{mg}$ protein. A. PD146176 markedly inhibited the production of linoleic acid-derived 13-HODE production $(* * * P<$ 0.001 vs. vehicle, $\mathrm{n}=3$ in each group). B. PD146176 had no effect on the production of arachidonic acid-derived 12-HETE and 15-HETE production. C. Gene silence of 15-LOX-1 markedly inhibited the production of linoleic acid-derived 13-HODE production (*** $P<0.001$ vs. vehicle, $\mathrm{n}=3$ in each group). D. Gene silence of $15-\mathrm{LOX}-2$ markedly inhibited the production of arachidonic acid-derived 15-HETE (*** $P<0.001$ vs. vehicle, $\mathrm{n}=3$ in each group).

A
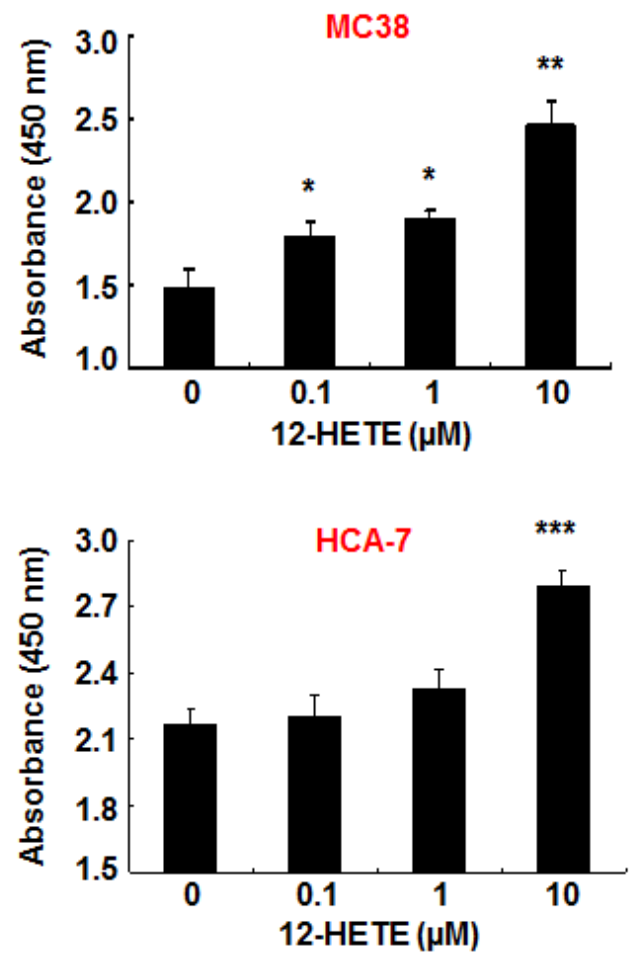

B
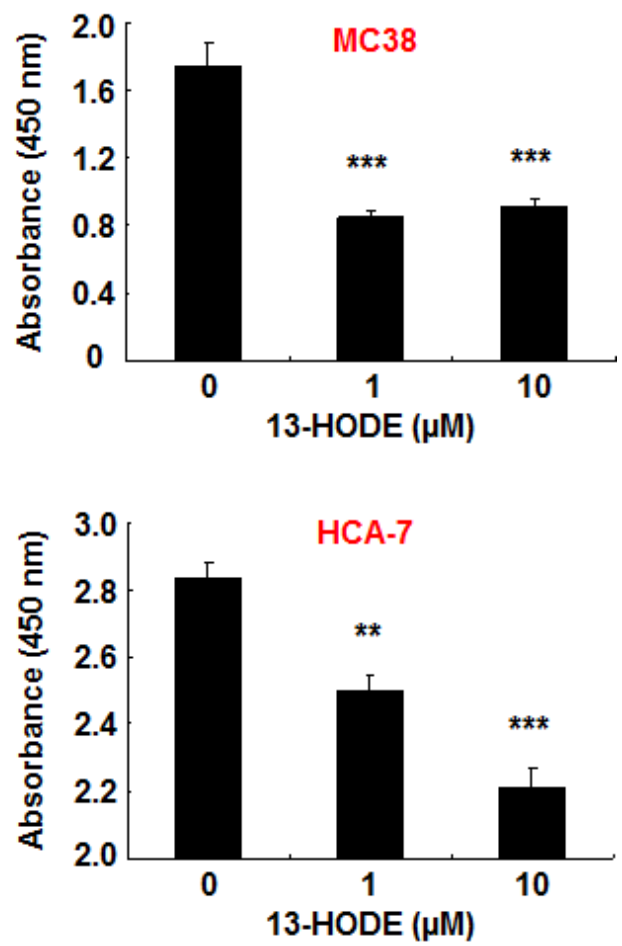

Figure 6: 12-HETE stimulated and 13-HODE inhibited murine and human colonic cancer cell proliferation. A. 12HETE stimulated both murine MC38 and human HCA-7 colon cancer cell proliferation $(* P<0.05, * * P<0.01, * * * P<0.01$. $\mathrm{N}=4)$. B. 13-HODE inhibited both murine MC38 and human HCA-7 colon cancer cell proliferation ( $* * P<0.01, * * * P<0.01 . \mathrm{N}=4$ in HCA-7 cells and $\mathrm{n}=6$ in MC38 cells). 
mouse colon cancer cell line (supplemental Figure S2). On the other hand, 13-HODE inhibited the proliferation of all tested mouse and human colon cancer cell lines (Figure 6 and supplemental Figure S2). 15-HETE had no effect on cell proliferation in all cell lines investigated (data not shown).

Human 15-LOX-1 mRNA levels were decreased in human colonic adenomas. Shureiqi et al reported that 13-HODE levels in human colorectal cancer mucosa and polyp mucosa were reduced compared to paired normal colon, while 12-HETE, 15-HETE and leukotriene B4 levels were comparable between normal colon and colorectal tumors[15]. Therefore we evaluate 15-LOX1 mRNA levels between adenomas with different sizes and their paired normal colon. As indicated in Figure 7, 15-LOX-1 mRNA levels were significantly reduced in both small and larger adenomas compared with normal colons $(* * * P<0.001$ vs. paired normal colon, $\mathrm{n}=7$ in each group).

\section{DISCUSSION}

The current studies were undertaken to investigate the potential role of $15-\mathrm{LOX}$ in colorectal tumorigenesis. The major findings include the following: (1) administration of PD146176, a commercially available, potent and selective inhibitor of $15-\mathrm{LOX}-1$, promoted human colonic HCA-7 tumor growth in association with decreased tumor levels of 13-HODE, which is consistent with the observation that human 15-LOX-1 suppresses the development and growth of human colorectal cancer; (2) PD146176 treatment inhibited murine colonic MC38 tumor growth in association with decreased tumor levels of both 12-HETE and 13-HODE; (3) 12/15-LOX deletion in the host tissue promoted MC38 tumor growth in association with decreased tumor 13-HODE alone; (4) PD146176 inhibited 13-HODE production in HCA-7



Figure 7: 15-LOX-1 mRNA levels were decreased in human colonic adenomas. Human colonic adenomas and paired normal colonic tissues were used for qPCR. 15-LOX-1 mRNA levels were decreased in both small $(<1 \mathrm{~cm})$ and larger $(>1 \mathrm{~cm})$ adenomas compared to paired normal colonic tissues $(* * * \mathrm{P}<0.001, \mathrm{n}=7)$. cells but inhibited 12-HETE production in MC38 cells; (5) 12/15-LOX deletion led to decreased production of 13-HODE in isolated intestinal macrophages; and (5) 12HETE stimulated and 13-HODE inhibited both murine and human colon cancer cell proliferation. Taken together, these results suggest that PD146176 might promote human colorectal tumor growth primarily due to inhibition of 13HODE production, but inhibit murine colorectal tumor growth as a result of inhibition of 12-HETE generation. The proposed mechanisms underlying rodent 12/15-LOX regulation of colorectal tumorigenesis are summarized in Figure 8.

Three murine 12/15-LOX isoforms exist: the leukocyte-type, the platelet-type, and the epidermis-type. The leukocyte-type $12 / 15$-LOX is the major isoform and possesses high enzymatic activity to generate 12HETE and 15-HETE from arachidonic acid and 13HODE from linoleic acid[22]. The leukocyte-type 12/15LOX is primarily expressed in macrophages, and mice with deletion of this isoform were used in our current studies[21, 23].

In a xenograft tumor model, tumors contain epithelial cells from exogenously injected cells and stroma cells from host. Tumor-stromal interactions play a key role in the development and progression of cancers. The solid tumor stromal microenvironment consists of infiltrating immune cells, fibroblasts, myofibroblasts, adipocytes, endothelial cells and pericytes as well as a variety of extracellular matrix components[24-26]. Macrophages are a major component of stroma and have

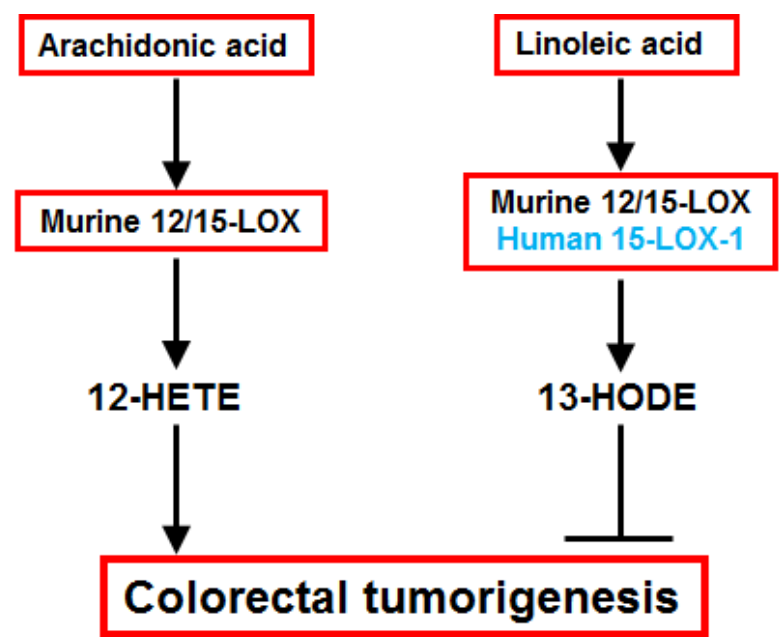

Figure 8: Proposed schema for arachidonic acid and linoleic acid metabolism-mediated colorectal tumorigenesis. Both rodent 12/15-LOX and human 15LOX-1 convert linoleic acid to 13-HODE to inhibit colorectal tumorigenesis. In addition, rodent $12 / 15-\mathrm{LOX}$ can also convert arachidonic acid to 12-HETE to promote colorectal tumorigenesis. The potential role of $12 / 15$-LOX in murine colonic cancer models should consider tumor cell type (human or mouse), the relative $12 / 15$-LOX activity in tumor cells and stroma, and the generation of 12-HETE and 13-HODE in the tumors. 
been reported to play an important role in colorectal tumorigenesis. Macrophages may release various chemicals and cytokines and chemokines that regulate tumor cell proliferation or apoptosis via paracrine action. In the current studies, we found that 12/15-LOX deletion led to marked decreases in the production of 13-HODE in mouse intestinal macrophages, suggesting that mouse macrophage 12/15-LOX can convert linoleic acid to 13HODE. As PD146176 also inhibited the production of 13HODE in human colon cancer HCA-7 cells, we postulate that PD146176-induced decreases in 13-HODE levels in HCA-7 tumors were due to inhibition of human HCA-7 tumor cell 15-LOX-1 activity and in mouse MC38 tumors, were due to inhibition of 12/15-LOX activity (particularly in macrophages).

In the cultured mouse colon cancer MC38 cells, PD146176 treatment inhibited the production of 12-HETE but not the production of 13-HDOE and 15-HETE. 12/15LOX deletion had no effect on the production of 12-HETE and 15-HETE in isolated mouse macrophages. Therefore, we suggest that the tumor promoting effect of host 12/15LOX deletion was due to inhibition of production of stromal cell 12/15-LOX-derived 13-HODE (Figure 6). On the other hand, our results indicate that PD146176 inhibited MC38 tumor growth due to inhibition of MC38 cell 12/15-LOX-derived 12-HETE, which is a potent mitogenic agent for MC38 cells (Figure 6).

Epidemiological studies in humans have suggested that high intake of linoleic acid can protect against tumorigenesis[27]. Both human 15-LOX-1 and rodent 12/15-LOX utilize linoleic acid as substrate to generate tumor suppressive 13-HODE. Peroxisome proliferatoractivated receptors (PPARs) are transcription factors that strongly influence molecular events in normal and cancer cells. PPARs can act as nuclear receptors for 13-HODE. PPAR- $\delta$ expression promotes colonic tumorigenesis[28], while activation of PPAR $\gamma$ inhibits cell proliferation and induces apoptosis in a retinoblastoma protein-dependent manner[29]. 13-HODE has been reported to inhibit the proliferation of colorectal cancer cells through downregulation of PPAR- $\delta$ signaling and promotion of PPAR- $\gamma$ activity[16, 30]. Linoleic acid is a major component of fat in commercially available rodent diet. For example, Basal Diet (Catalog \# 7024,TestDiet, St. Louis, MO) contains $3.34 \%$ linoleic acid, the major component of fat in the diet. Therefore, there is enough substrate for $12 / 15 \mathrm{LOX}$ to generate 13-HODE in vivo.

Although investigation of the role of $12 / 15$-LOX in colorectal tumorigenesis is limited and controversial in animal models[18, 19], studies in human colorectal tumorigenesis have consistently indicated that $15-\mathrm{LOX}-1$ and its linoleic acid metabolite, 13-HODE, inhibit tumor growth[14, 16, 31]. 13-HODE inhibits human colorectal carcinoma cell proliferation and overexpression of 15LOX-1 inhibits human colon cancer cell proliferation and induces apoptosis[32-34]. Shureiqi et al. recently reported that 13-HODE levels were decreased in colorectal polyp mucosa and in colorectal cancer mucosa compared with paired normal colon, while the levels of other lipoxygenase-mediated metabolites including 12- or 15-hydroxyeicosatetraenoic acid (12-HETE, 15-HETE) or leukotriene B4 levels were comparable between normal colon and colorectal tumors[15]. In addition, induction of 15-LOX-1 expression and 13-HODE production has been reported to contribute to colorectal cancer cell apoptosis induced by NSAIDs[35]. Even expression of human 15LOX-1 in mouse colonic epithelial cells can suppress colonic tumorigenesis[19]. Although eicosanoid profiling was not available in this study, it is possible that 13-HODE production increased in these cells, because linoleic acid, the substrate of human 15-LOX-1, is robust in rodent food as described above.

In summary, 15-LOX-1 and its linoleic acid metabolite, 13-HODE, inhibit human colorectal tumorigenesis. However, the effect of rodent 12/15 LOX on colorectal tumorigenesis is complicated and is determined by several factors: tumor cell type (human or mouse), tumor cell 12/15 LOX level, host 12/15-LOX activity, relative production of arachidonic acid and linoleic acid metabolites, and availability of substrate.

\section{METHODS}

\section{Ethics Statement}

Investigation has been conducted in accordance with the ethical standards and according to the Declaration of Helsinki and according to national and international guidelines and has been approved by the Institutional Animal Care and Use Committee of Vanderbilt University.

\section{Animals}

12/15-LOX knockout mice (C57BL/6, stock number 002778) were purchased from Jackson Laboratories. Athymic nude mice were purchased from Harlan Sprague Dawley (male, 8 week old).

\section{Cell culture and cell proliferation assays}

Mouse colon adenocarcinoma MC38 cells (C57BL/6) and CT26 cells (BALB/c), human colon carcinoma HCA-7 cells, KM12 cells and DLD cells were grown in RPMI-1640 supplemented with $4,500 \mathrm{mg} / \mathrm{L}$ glucose, $2 \mathrm{mM}$ L-glutamine, $10 \%$ fetal bovine serum, $100 \mathrm{U} / \mathrm{ml}$ penicillin, and $100 \mu \mathrm{g} / \mathrm{ml}$ streptomycin in $5 \%$ $\mathrm{CO}_{2}$ and $95 \%$ air at $37^{\circ} \mathrm{C}[36]$. The cells were seeded in a 96-well plate $\left(1 \times 10^{4}\right.$ cells in $200 \mu 1$ medium) for 16 hours, then starved for 24 hours in medium containing 
$0.1 \%$ fetal bovine serum, and various concentrations of agents (12-HETE, 15-HETE and 13-HODE) were added for additional 4 hours. Then BrdU solution was added to each well for 4 hours. Analysis was performed according to the manufacturer's protocol (BrdU cell proliferation assay kit, Cat\#6813, Cell Signaling).

\section{Primary tumor growth}

For mouse colon adenocarcinoma MC38 tumor experiments, male wild type and 12/15-LOX-/- mice (C57BL/6, 8 weeks old) were given 2 dorsal s.c. injections of MC38 cells ( $5 \times 10^{5}$ cells per site). Wild type mice were treated with vehicle (water) or 12/15-LOX inhibitor, PD146176 (5 mg/kg/day, mini-pump, 2004) and sacrificed after 18 day of growth. Tumors were weighed at the end of experiment. For human colon carcinoma experiments, athymic nude mice were given 2 dorsal s.c. injections of HCA-7 cells $\left(3.35 \times 10^{6}\right.$ cells per site $)$ with administration of vehicle (water) or PD146176 (5 mg/kg/day, minipump, 2004) and sacrificed after 4 week of growth. Under anesthesia with Nembutal (60 mg/kg, i.p.), the tumor was dissected, weighed, and snap-frozen in liquid $\mathrm{N}_{2}$ for eicosanoid measurement and other analysis or fixed in FPAS for immunohistochemistry[37].

\section{Isolation of intestinal monocytes/macrophages}

Intestinal monocytes/macrophages in singlecell suspensions were enriched using mouse CD11b Microbeads and MACS columns (Miltenyi Biotec) following the manufacturer's protocol.

\section{Enzyme activity assays}

Either isolated monocytes/macrophages or cultured cells were incubated in serum-free RPMI-1640 medium containing $60 \mu \mathrm{M}$ arachidonic acid or linoleic acid for 1 hour, medium was harvested for eicosanoid measurements. Protein concentration was determined by using Pierce BCA protein assay kit (Cat\#23225, Thermo Scientific). Enzyme activity was expressed as $\mathrm{pM} / \mathrm{min} / \mathrm{mg}$ protein.

\section{RNA interference}

siRNAs and negative control siRNA (mock) included ALOX12-human (Cat\# 4392420), ALOX15human (15-LOX-1, cat\#4392420), ALOX15B-human (15-LOX-2, cat\# 4392420), Alox15-mouse (12/15-LOX, cat\# 4390771) and Silencer Selective Control NO.1 siRNA (cat\# 4390843) were purchased from Invitrogen. Cells were seeded at a density of $3 \times 10^{5}$ cells per well on 6-well plates and grown in $10 \% \mathrm{FBS} / \mathrm{DMEM}$ without antibiotics. After $24 \mathrm{~h}$, the cells were transfected with siRNA using
Lipofectamine ${ }^{\circledR}$ RNAiMAX (Invitrogen) according to the manufacturer's instruction. Briefly, 25 pmol siRNA and $7.5 \mu$ RNAiMAX (Invitrogen) were diluted in Opti-MEM (Invitrogen) without serum and antibiotics. After mixing for $15 \mathrm{~min}$ at room temperature, the mixture was added to a final volume of $1.5 \mathrm{ml}$, and the cells were incubated at $37^{\circ} \mathrm{C}$ in a humidified incubator. After $24 \mathrm{~h}$ incubation, the interference mixture was removed, and complete culture medium was added. The enzyme activity assays were performed in the cells at $72 \mathrm{~h}$ after siRNA transfection as described above.

\section{Measurement of eicosanoids}

Tumor tissue and culture medium eicosanoid profiling was measured by gas chromatographic/negative ion chemical ionization mass spectrometric assays using stable isotope dilution, as described previously[20, 38].

\section{RNA isolation and quantitative real-time PCR}

Total RNA was isolated from tumors using TRIzol reagents (Invitrogen) according to the manufacturer's instructions. Quantitative PCR was performed using TaqMan real time PCR machine (7900HT, Applied Biosystems). The Master Mix and gene probes were also purchased from Applied Biosystems. The primers used for human ALOX15 (Hs00993766) and $\beta$-actin (Hs99999903) were from Applied Biosystems.

\section{Huuman tissues}

The human samples used in this study were from the Tennessee Colorectal Polyp Study (TCPS) protocol that was approved by the Institutional Review Board of Vanderbilt Medical Center, and written, informed consent was obtained from all participants.

\section{Statistics}

All values are presented as means, with error bars representing \pm s.e.m. ANOVA and Bonferroni $t$ tests were used for statistical analysis, and differences were considered significant when $P<0.05$.

\section{ACKNOWLEDGEMENTS}

This work was supported by National Institute of Health grants CA-122620 (to M.-Z.Z.), DK-51265 and DK-95785 (to M.-Z.Z.), CA97386 (to W. Zheng), and by National Natural Science Foundation of China (81472705 to Li Jiang). We would like to thank Dr. Raymond C Harris for his helpful comments. 


\section{REFERENCES}

1. Wang D and Dubois RN. Epoxyeicosatrienoic acids: a double-edged sword in cardiovascular diseases and cancer. J Clin Invest. 2012; 122(1):19-22.

2. Gomez A, Nekvindova J, Travica S, Lee MY, Johansson I, Edler D, Mkrtchian S and Ingelman-Sundberg $\mathrm{M}$. Colorectal cancer-specific cytochrome P450 2W1: intracellular localization, glycosylation, and catalytic activity. Mol Pharmacol. 2010; 78(6):1004-1011.

3. Wang D and Dubois RN. Eicosanoids and cancer. Nat Rev Cancer. 2010; 10(3):181-193.

4. Panigrahy D, Edin ML, Lee CR, Huang S, Bielenberg DR, Butterfield CE, Barnes CM, Mammoto A, Mammoto T, Luria A, Benny O, Chaponis DM, Dudley AC, Greene ER, Vergillio JA, Pietramaggiori G et al. Epoxyeicosanoids stimulate multiorgan metastasis and tumor dormancy escape in mice. J Clin Invest. 2012; 122(1):178-191

5. Castellone MD, Teramoto H, Williams BO, Druey KM and Gutkind JS. Prostaglandin E2 promotes colon cancer cell growth through a Gs-axin-beta-catenin signaling axis. Science. 2005; 310(5753):1504-1510.

6. Barresi V, Vitarelli E, Tuccari G and Barresi G. Correlative study of microvessel density and 5-lipoxygenase expression in human sporadic colorectal cancer. Arch Pathol Lab Med. 2008; 132(11):1807-1812.

7. Barresi V, Grosso M, Vitarelli E, Tuccari G andBarresi G. 5-Lipoxygenase is coexpressed with Cox-2 in sporadic colorectal cancer: a correlation with advanced stage. Dis Colon Rectum. 2007; 50(10):1576-1584.

8. Melstrom LG, Bentrem DJ, Salabat MR, Kennedy TJ, Ding XZ, Strouch M, Rao SM, Witt RC, Ternent CA, Talamonti MS, Bell RH and Adrian TA. Overexpression of 5-lipoxygenase in colon polyps and cancer and the effect of 5-LOX inhibitors in vitro and in a murine model. Clin Cancer Res. 2008; 14(20):6525-6530.

9. Cheon EC, Khazaie K, Khan MW, Strouch MJ, Krantz SB, Phillips J, Blatner NR, Hix LM, Zhang M, Dennis KL, Salabat MR, Heiferman M, Grippo PJ, Munshi HG, Gounaris E and Bentrem DJ. Mast cell 5-lipoxygenase activity promotes intestinal polyposis in APCDelta468 mice. Cancer Res. 2011; 71(5):1627-1636.

10. Shin VY, Jin HC, Ng EK, Sung JJ, Chu KM and Cho $\mathrm{CH}$. Activation of 5-lipoxygenase is required for nicotine mediated epithelial-mesenchymal transition and tumor cell growth. Cancer Lett. 2010; 292(2):237-245.

11. Saini MK and Sanyal SN. PTEN regulates apoptotic cell death through PI3-K/Akt/GSK3beta signaling pathway in DMH induced early colon carcinogenesis in rat. Exp Mol Pathol. 2012; 93(1):135-146.

12. Honn KV, Tang DG, Gao X, Butovich IA, Liu B, Timar J and Hagmann W. 12-lipoxygenases and 12(S)-HETE: role in cancer metastasis. Cancer Metastasis Rev. 1994; 13(34):365-396.
13. Gong Z, Hebert JR, Bostick RM, Deng Z, Hurley TG, Dixon DA, Nitcheva D and Xie D. Common polymorphisms in 5-lipoxygenase and 12-lipoxygenase genes and the risk of incident, sporadic colorectal adenoma. Cancer. 2007; 109(5):849-857.

14. Shureiqi I, Wojno KJ, Poore JA, Reddy RG, Moussalli MJ, Spindler SA, Greenson JK, Normolle D, Hasan AA, Lawrence TS and Brenner DE. Decreased 13-S-hydroxyoctadecadienoic acid levels and 15-lipoxygenase-1 expression in human colon cancers. Carcinogenesis. 1999; 20(10):1985-1995.

15. Shureiqi I, Chen D, Day RS, Zuo X, Hochman FL, Ross WA, Cole RA, Moy O, Morris JS, Xiao L, Newman RA, Yang $P$ and Lippman SM. Profiling lipoxygenase metabolism in specific steps of colorectal tumorigenesis. Cancer Prev Res (Phila). 2010; 3(7):829-838.

16. Shureiqi I, Jiang W, Zuo X, Wu Y, Stimmel JB, Leesnitzer LM, Morris JS, Fan HZ, Fischer SM and Lippman SM. The 15-lipoxygenase-1 product 13-S-hydroxyoctadecadienoic acid down-regulates PPAR-delta to induce apoptosis in colorectal cancer cells. Proc Natl Acad Sci USA. 2003; 100(17):9968-9973.

17. Kelavkar UP, Glasgow W, Olson SJ, Foster BA and Shappell SB. Overexpression of 12/15-lipoxygenase, an ortholog of human 15-lipoxygenase-1, in the prostate tumors of TRAMP mice. Neoplasia. 2004; 6(6):821-830.

18. Kerjaschki D, Bago-Horvath Z, Rudas M, Sexl V, Schneckenleithner C, Wolbank S, Bartel G, Krieger S, Kalt R, Hantusch B, Keller T, Nagy-Bojarszky K, Huttary N, Raab I, Lackner K, Krautgasser K et al: Lipoxygenase mediates invasion of intrametastatic lymphatic vessels and propagates lymph node metastasis of human mammary carcinoma xenografts in mouse. J Clin Invest. 2011; 121(5):2000-2012.

19. Zuo X, Peng Z, Wu Y, Moussalli MJ, Yang XL, Wang Y, Parker-Thornburg J, Morris JS, Broaddus RR, Fischer SM and Shureiqi I. Effects of gut-targeted 15-LOX-1 transgene expression on colonic tumorigenesis in mice. J Natl Cancer Inst. 2012; 104(9):709-716.

20. Zhang MZ, Xu J, Yao B, Yin H, Cai Q, Shrubsole MJ, Chen $\mathrm{X}$, Kon V, Zheng W, Pozzi A and Harris RC. Inhibition of 11 beta-hydroxysteroid dehydrogenase type II selectively blocks the tumor COX-2 pathway and suppresses colon carcinogenesis in mice and humans. J Clin Invest. 2009; 119(4):876-885.

21. Kriska T, Cepura C, Magier D, Siangjong L, Gauthier $\mathrm{KM}$ and Campbell WB. Mice lacking macrophage 12/15-lipoxygenase are resistant to experimental hypertension. Am J Physiol Heart Circ Physiol. 2012; 302(11):H2428-2438.

22. Burger F, Krieg P, Marks F and Furstenberger G. Positionaland stereo-selectivity of fatty acid oxygenation catalysed by mouse (12S)-lipoxygenase isoenzymes. Biochem J. 2000; 348 Pt 2:329-335.

23. Sun D and Funk CD. Disruption of 12/15-Lipoxygenase 
Expression in Peritoneal Macrophages. ENHANCED UTILIZATION OF THE 5-LIPOXYGENASE PATHWAY AND DIMINISHED OXIDATION OF LOW DENSITY LIPOPROTEIN. J Biol Chem. 1996; 271(39):24055-24062.

24. McAllister SS and Weinberg RA. Tumor-host interactions: a far-reaching relationship. J Clin Oncol. 2010; 28(26):40224028.

25. Junttila MR and de Sauvage FJ. Influence of tumour microenvironment heterogeneity on therapeutic response. Nature. 2013; 501(7467):346-354.

26. Gajewski TF, Schreiber H and Fu YX. Innate and adaptive immune cells in the tumor microenvironment. Nat Immunol. 2013; 14(10):1014-1022.

27. Horrobin DF and Ziboh VA. The importance of linoleic acid metabolites in cancer metastasis and in the synthesis and actions of 13-HODE. Adv Exp Med Biol. 1997; 433:291294.

28. Park BH, Vogelstein B and Kinzler KW. Genetic disruption of PPARdelta decreases the tumorigenicity of human colon cancer cells. Proc Natl Acad Sci USA. 2001; 98(5):25982603.

29. Fajas L, Egler V, Reiter R, Miard S, Lefebvre AM and Auwerx J. PPARgamma controls cell proliferation and apoptosis in an RB-dependent manner. Oncogene. 2003; 22(27):4186-4193.

30. Zuo X, Wu Y, Morris JS, Stimmel JB, Leesnitzer LM, Fischer SM, Lippman SM and Shureiqi I. Oxidative metabolism of linoleic acid modulates PPAR-beta/delta suppression of PPAR-gamma activity. Oncogene. 2006; 25(8):1225-1241.

31. Shureiqi I, Wu Y, Chen D, Yang XL, Guan B, Morris JS, Yang P, Newman RA, Broaddus R, Hamilton SR, Lynch B, Fischer SM and Lippman. The critical role of 15-lipoxygenase-1 in colorectal epithelial cell terminal differentiation and tumorigenesis. Cancer Res. 2005; 65(24):11486-11492.

32. Nixon JB, Kim KS, Lamb PW, Bottone FG and Eling TE. 15-Lipoxygenase-1 has anti-tumorigenic effects in colorectal cancer. Prostaglandins Leukot Essent Fatty Acids. 2004; 70(1):7-15.

33. Cimen I, Astarci E and Banerjee S. 15-lipoxygenase-1 exerts its tumor suppressive role by inhibiting nuclear factor-kappa B via activation of PPAR gamma. J Cell Biochem. 2011; 112(9):2490-2501.

34. Cimen I, Tuncay S and Banerjee S. 15-Lipoxygenase-1 expression suppresses the invasive properties of colorectal carcinoma cell lines HCT-116 and HT-29. Cancer Sci. 2009; 100(12):2283-2291.

35. Shureiqi I, Chen D, Lee JJ, Yang P, Newman RA, Brenner DE, Lotan R, Fischer SM and Lippman SM. 15-LOX-1: a novel molecular target of nonsteroidal anti-inflammatory drug-induced apoptosis in colorectal cancer cells. J Natl Cancer Inst. 2000; 92(14):1136-1142.

36. Jiang L, Yang S, Yin H, Fan X, Wang S, Yao B, Pozzi
A, Chen X, Harris RC and Zhang MZ. Epithelial 11betahydroxysteroid dehydrogenase type II deletion inhibits Apc $+/$ min mouse tumorigenesis via COX-2 pathway inhibition and induction of G1 cell cycle arrest. Mol Cancer Res. 2013; 11(9): 1040-1050.

37. Zhang MZ, Yao B, Cheng HF, Wang SW, Inagami $\mathrm{T}$ and Harris RC. Renal cortical cyclooxygenase 2 expression is differentially regulated by angiotensin II AT(1) and AT(2) receptors. Proc Natl Acad Sci U S A. 2006; 103(43):1604516050 .

38. Brady AE, Jones CK, Bridges TM, Kennedy JP, Thompson AD, Heiman JU, Breininger ML, Gentry PR, Yin H, Jadhav SB, Shirey JK, Conn PJ and Lindsley CW. Centrally active allosteric potentiators of the M4 muscarinic acetylcholine receptor reverse amphetamine-induced hyperlocomotor activity in rats. J Pharmacol Exp Ther. 2008; 327(3):941953. 